\title{
Foreword by the Chairman of the EISCAT Council
}

\author{
Mike Lockwood \\ Rutherford Appleton Laboratory, Oxfordshire, UK
}

This volume contains papers presented at the 10th biennial International EISCAT workshop, held in Tokyo, Japan, in July 2001. The quality and excitement of the papers clearly demonstrate that the vitality and diversity of the science made possible by these radars is stronger than ever and that the facilities have maintained their pre-eminence in the field. For more than 20 years, the EISCAT radars have been producing world-leading data for research into space plasmas. The majority of the observations are of the ionosphere; however, because the behaviour of the ionospheric plasma is strongly linked by collisions to the upper neutral atmosphere and, via electomagnetic coupling and auroral particle precipitation, to the magnetosphere, EISCAT is able to study both these regions of geospace as well. The location of the EISCAT Svalbard Radar under the cusp allows studies of how Earth's magnetosphere-ionosphere system interacts with the solar wind; the locations of the mainland systems are ideal for studying the internal dynamics of the magnetospheric tail. In addition, EISCAT is unique in having a high-power HF modification ("heater") facility supported by an incoherent scatter radar. In fact, in addition to having both the UHF and VHF radars as diagnostics, the heater field-of-view is also monitored by a digital ionosonde and the CUTLASS HF radar system as well as optical instrumentation and an imaging riometer. This combination really does turn the ionosphere over Tromsø into a natural laboratory for studying the mechanisms and behaviour of large-scale plasma. As an example, studies of "artificial aurora" induced by the heater have given new insights into plasma-wave interactions. In addition to this broad diversity of science, the interplanetary scintillation technique has been applied to EISCAT with great success, making it possible to observe uniquely close to the Sun and, consequently, to study the acceleration of the solar wind. Further developments will allow yet more diversification into areas such as space debris and interplanetary particles.

EISCAT may have been operating for more than 20 years, but in recent years it has been subject to a major expansion and renovation. The second, 42-metre antenna for the Svalbard radar is now operational, allowing field-aligned profiles of the cusp ionosphere to be monitored continuously while the 32-metre antenna can be deployed in a variety of modes to define the surrounding conditions. This is opening up novel studies of cusp precipitation and ion outflow and of the mysterious and intriguing anomalous coherent echoes. The mainland radars have enjoyed a major upgrade, with a number of tremendous benefits: the availability of enhanced signal processing power allows the achievement of even better range and time resolutions; the system now supports a range of new alternating code experiments with advanced new coding schemes (for example for studies of auroral arcs and the D-region); ultimately the upgrades will allow higher transmitter powers to be used for the UHF with consequent increases in range and space and time resolutions. Interplanetary scintillation work has been protected from the intrusion of noise transmissions by new hardware for $1.4 \mathrm{GHz}$ operations and studies of polarisation are now coming on-stream with the promise of remote sensing of the magnetic field, which should prove to be a crucial advance for space-weather applications.

Solar-terrestrial physics is now a multi-point, multiinstrument, multi-technique scientific discipline. Powerful as the EISCAT radars are as diagnostics of fundamental space plasma processes, their full value becomes apparent when they are used with other instrumentation. In the past two years, one of the prime examples of this has been the highly successful co-ordination of operations with the European Space Agency's Cluster spacecraft and similar co-ordinated experiments with Geotail, FAST and TIMED are underway. Furthermore, the location of the ESR on Svalbard means that there are many and frequent conjunctions with the various DMSP (Defense Meteorological Satellite Program) satellites and the combination of these data is very powerful indeed. In addition, new supporting ground-based diagnostics have been put in place. For example the spectrographs at Troms $\emptyset$ and Svalbard will allow important new studies of protoninduced aurora in combination with the radars; in addition, the SPEAR HF radar/heater on Svalbard will come on stream in the near future, making possible a whole new range of experiments.

The papers in this volume use the full power and diversity of the EISCAT systems to study a wide selection of scientific 
topics:

- The data are used to characterise electron precipitation and ion-neutral heating in the upper atmosphere. Dayside heating and precipitation reveals the influence of nightside phenomena and substorms in particular. The variations of the precipitation spectrum within the substorm have also been studied using EISCAT in conjunction with satellites and imaging riometer data.

- The first ever satellite detection of artificially-excited Alfvénic ULF waves, produced by the heater, is presented. The downward electron flux data in this event contain the first observations of electrons undergoing acceleration within the Ionospheric Alfvén Resonator due to parallel electric fields associated with an artificially stimulated Alfvén wave. EISCAT has also been used to study the relative contributions of spatial conductivity gradients and the divergence of electric fields to the field-aligned currents associated with auroral arcs.

- Cusp observations are used with satellite data to determine the complex morphology of magnetopause reconnection, in particular to study the interplanetary conditions that allow interesting intervals when both subsolar and lobe reconnection can occur simultaneously.

- An analysis of the causes of storm-time enhancements of the high latitude ionosphere is presented.

- At lower altitudes, intriguing new observations showing merging sub-structure in sporadic-E layers are presented. Tidal temperature oscillations near the mesopause are detected using multipoint meteor radar observations.

- The effects of both natural and induced ionospheric irregularities on HF propagation are studied in coordinated experiments. Particularly encouraging are the new techniques that are presented here. These increase the diversity and scientific power of the EISCAT systems.

- Studies aimed at understanding the occurrence of echoes in HF coherent radars are important in developing the techniques for combining incoherent and coherent scatter data. For example, such work is leading to a better understanding of how the data can be used to identify important features of the magnetosphereionosphere system, such as the open-closed field line boundary.

- Similarly, studies aimed at understanding the occurrence of scintillation echoes in the solar wind are important in developing the interplanetary scintillation technique (IPS). New methods have been developed to allow studies of low-frequency Alfvén waves in the solar wind. Conclusions from IPS techniques have been checked using in situ heliospheric data from the Wind and Ulysses satellites.

- Conductivities for high and middle latitudes at 70$100 \mathrm{~km}$ have been modeled from 11 years' VHF radar data and this will allow new studies of the properties of Schumann resonances.

- The heater has been used to generate irregularities which allow astonishing definition of ULF waves in HF coherent scatter data. Evidence is presented here which links these waves to non-Maxwellian precipitation seen in satellite data.

- A new D-region experiment with 600-m resolution is unveiled.

This brief summary is sufficient to demonstrate that the both the EISCAT facility and its scientific user community are working outstandingly well to generate exciting new science. Personally, I have been involved with EISCAT for sufficiently long to remember the excitement in the community when the first data were discussed at the first workshop. It is wonderful to see that some of my contemporaries from 20 years ago are still very active in the field. However, I am sure that they will agree with me when I say that it is particularly gratifying to note the large number of younger scientists and students who contributed so much to this, the 10th workshop. However, whatever their age, all the scientists who use the radars will join me in thanking the director and staff of EISCAT for the provision of these superb facilities and for their efforts that have kept them at the cutting edge. They will also, like me, thank the organising committee of this wonderful workshop and the editorial office of Annales Geophysicae for producing this addition to the series of EISCAT workshop special issues which, in every sense, uphold the tradition of excellence. 